\title{
Fluctuations and correlations in an individual-based model of biological coevolution
}

\author{
R K P Zia† \\ $\dagger$ Center for Stochastic Processes in Science and Engineering, Department of Physics, \\ Virginia Polytechnic Institute and State University, Blacksburg, Virginia 24061-0435, \\ USA \\ $\ddagger$ Fachbereit Physik, Universität Duisburg-Essen, 45117 Essen, Germany \\ I School of Computational Science and Information Technology, Center for Materials \\ Research and Technology, and Department of Physics, Florida State University, \\ Tallahassee, Florida 32306-4120, USA \\ E-mail: rkpzia@vt.edu, rikvold@csit.fsu.edu
}

\begin{abstract}
We extend our study of a simple model of biological coevolution to its statistical properties. Staring with a complete description in terms of a master equation, we provide its relation to the deterministic evolution equations used in previous investigations. The stationary states of the mutationless model are generally well approximated by Gaussian distributions, so that the fluctuations and correlations of the populations can be computed analytically. Several specific cases are studied by Monte Carlo simulations, and there is excellent agreement between the data and the theoretical predictions.
\end{abstract}

Submitted to: $J P A$

PACS numbers: 05.40.-a 87.23.Kg 05.65.+b 


\section{Introduction}

The dynamics of populations and species in the context of biological or ecological systems have attracted considerable attention in the community of statistical physicists in the last decade. Though there has been much progress [1, 2, it is rare that the behavior of macroscopic populations can be predicted, even for "simple models," from a set of stochastic rules for an individual's propensity to survive and/or reproduce. The difficulties can be traced not only to the presence of many degrees of freedom, with complex internal interactions (e.g., mutualistic or predator-prey), but also to the non-trivial couplings to external reservoirs (such as energy or food). As a result, even if we only focus on systems in stationary states, we must recognize that these are non-equilibrium steady states, so that the well-known methods of equilibrium thermodynamics should not be blindly applied. At present, given the absence of a universally applicable framework of non-equilibrium statistical mechanics, progress is made through understanding "one system at a time." Within this context, we recently studied a model of coevolution [3, 4, based on the one ("tangled nature") introduced by Hall, Christensen, and collaborators [5, 6, 7]. The motivations behind these studies are varied. An early model for coevolution and speciation, introduced by Bak and Sneppen [8], consists of competing species, according to a preassigned notion of "fitness." Speciation arises by imposing a crude version of "mutation," i.e., letting the least fit species (as well as some of their "neighbors") be replaced by new species with different, randomly chosen fitness. Despite their simplicity, such models appear to settle into a steady state which exhibits avalanches of extinctions. Though they are hailed as showing a link between Darwin's principle of "survival of the fittest" and Gould's notion of "punctuated equilibria" [9, 10, 11, these models are thought to be too simplistic in at least two aspects. Firstly, mutation and selection act on individuals of a populations, rather than on entire species at once. Secondly, whether a species is "fit" is not a static notion, but rather depends on what other species are present in the ecosystem.

To address both issues, Hall, et.al. [5, 6, 7] recently introduced an individual-based model with a dynamically evolving "fitness landscape." The "ecosystem" consists of individuals, each of which is said to belong to a "species" identified by a "genome" represented by a string of bits. Mutations are built in through the random flipping of bits, at a constant slow rate, in the genomes of newborn individuals. In earlier models of speciation, an individual's fitness, i.e., its reproduction probability, is purely a function of the bit string and fixed for all time. Here, the reproduction probability depends on the relative abundance of all other species, so that "fitness" becomes a dynamic concept. The "interspecies interactions" are, by contrast, comparatively static in nature and, for simplicity, are introduced via fixed quantities associated with pairs of genotypes. Thus, the model accounts only for whether (an individual of) a species is beneficial or detrimental for another species, regardless of the presence of any other species. Again for simplicity, all species reproduce asexually, with identical fecundities. Simulations reveal

several interesting behaviours, including the presence of long-lived states separated by 
bursts of high activity - "punctuated equilibria." Unfortunately, this model proved too complex for analytic understanding, and only phenomenological descriptions have been advanced to date.

In an effort to gain some insight into how its remarkable properties arise, we considered a variation of this model, with simpler interspecies interactions, which enables us both to carry out much longer simulations and to perform linear stability analysis [3, 4]. In addition to observing a self-similar picture of intermittency or "punctuated equilibria" over several decades of generations, we found that standard measures of diversity display $1 / f$ noise in their power spectral densities. This property is quite consistent with another finding: The life-time distribution of the long-lived states approximately follows a inverse-square power law [12. More detailed investigations of the very long-lived states, which typically consist of a community of a handful of dominant species along with a "cloud of mutants," reveal the reason behind their longevity. Essentially none of the closest mutants of the main species in such a community are "dangerous," in that their interactions with the parent species inhibit their exponential growth. In other words, the original community is (linearly) stable against invasion by its most closely related mutants. Only a small fraction of the nextclosest mutants (with genes differing from a dominant species by two bits) are dangerous, leading to the eventual demise of the "quasi-steady" state. Despite the discovery of these connections, full analytic understanding is still beyond our grasp. In particular, since the theoretical analyses were based entirely on a heuristic, deterministic ("mean-field") equation of motion, it is unlikely that they can account for the most intriguing behaviour stemming from a stochastic dynamics.

In the present paper, we address some of the issues associated with a fully stochastic description. Starting from a master equation which governs the evolution of all the details ("microscopics") of the model, we first demonstrate that the deterministic equation in 4] emerges, provided all correlations are ignored. However, it is no easy task to find a quantitative understanding describing the quasi-steady states (QSS), in which the system appears to be stationary but actually has long, finite lifetimes. One complication lies with the inherent metastability aspect of a QSS. Another is that, due to mutations, the populations in a QSS consist of two components: a handful of dominant species (with at least several hundred individuals, in the specific simulations we ran) and a larger number of minor species (with much less than one hundred individuals). Our approach to the solution is a two-step process. First, for each QSS, we develop a full understanding of a corresponding "truly stationary state" (TSS), i.e., one with only the dominant species. Each TSS is associated with an $\mathcal{N}$-species fixed point discussed in [4] and can be accessed by setting the mutation rate, $\mu$, to zero. The second step is to account for $O(\mu)$ effects, by including a limited "cloud of mutants." Needless to say, a careful definition of such a community will be necessary before any analytic progress is possible. Thus, we will only take the first step here, deferring the more complex problem to a later publication. Beyond that, our eventual goal is to predict the more fascinating phenomena, such as power-law distribution of QSS lifetimes, "punctuated equilibria" 
(or intermittency), and $1 / f$ noise.

In the next section, for completeness and the readers' convenience, we briefly review the specifications of the model. The following section is devoted to the master equation and the derivation of the deterministic evolution equation used in [4. Fluctuations and correlations in a TSS, as well as comparisons to simulations in specific cases, are the focus of sections 4 and 5 . Conclusions and an outlook can be found in section 6. The Appendix is devoted to some of the technical details.

\section{Model specification and algorithm}

The model we considered [4] is a simplified version of the one introduced in [5, 6, 7]. It consists of a population of individuals, each of which is associated with a string $L$ of bits (0 or 1 ), representing a "genome" of $L$ "genes." The bit-strings, or genotypes, are labeled by integers $I$, which lie between 1 and $\mathcal{N}_{\max }=2^{L}$. For simplicity, we will use the term "species" to distinguish individuals with different bit-strings or genotypes. The population evolves asexually in discrete time steps $(t=0,1, \ldots)$, which may be thought of as "years" or "generations." In our model, all individuals of a generation die when those of the next generation are "born" (as in, e.g., aphids). Thus, for any particular run (or "history"), the system is fully specified by the set of integers $n_{I}(t)$ $\left(I=1, \ldots, \mathcal{N}_{\max }\right)$ representing the number of individuals of genotype $I$ in generation $t$. In cases where the explicit index $I$ is not necessary, we will use the "vector" notation:

$$
\vec{n}(t) \equiv\left\{n_{1}(t), \ldots, n_{\mathcal{N}_{\max }}(t)\right\} .
$$

To model competition and interspecies interactions, we let an individual die with some non-vanishing probability before it reproduces, e.g., salmon that die in the oceans. All survivors then give birth to $F$ offspring, which constitute the next generation. Competition for resources (e.g., space, energy, food) is often introduced via a Verhulst 13. factor, which also plays the role of preventing unlimited growth and enters typically via a ratio $N_{\text {tot }}(t) / N_{0}$. Here, $N_{0}$ is a parameter representing the "carrying capacity" of the "ecosystem," and

$$
N_{\text {tot }}(t) \equiv \sum_{I} n_{I}(t)
$$

is the total population at time $t$. With a "healthy" fecundity $(F)$, the population is unlikely to "collapse" $\left(N_{\text {tot }}=0\right)$. Instead, $N_{\text {tot }}(t)$ is rarely far from $N_{0}$. Interspecies interactions are modeled by a matrix $\mathbf{M}$, the element $M_{I}^{J}$ being the effect of the species $J$ on species $I$. For reasons provided in [4], we set $M_{I}^{I}=0$ and choose random offdiagonal elements from a uniform distribution over $[-1,1]$. In all our simulations, $\mathbf{M}$ is fixed at $t=0$ and does not evolve in time. If both $M_{I}^{J}$ and $M_{J}^{I}$ are positive, the two species are said to be "mutualistic," and if they are of opposite signs, we have a predator-prey relationship. Not surprisingly, populations with both elements being negative are extremely unstable. Finally, the probability of an individual of species $I$ 
to survive to reproduction is specified by [4]

$$
P(I ; \vec{n}(t))=\frac{1}{1+\exp \left[-M_{I}^{J} n_{J}(t) / N_{\text {tot }}(t)+N_{\text {tot }}(t) / N_{0}\right]},
$$

which is often shortened to just $P(I)$. Here, as in the rest of the paper, we use the Einstein summation convention, i.e., a repeated index (e.g., $J$ in $M_{I}^{J} n_{J}$ ) is summed over (from 1 to $\mathcal{N}_{\max }$, in general). As will be clear below, where we deal with systems with large $N_{0}$ ("macroscopic," though not necessarily in the sense of typical thermodynamic systems), quantities with a single subscripted index (e.g., $n_{J}$ ) are generally of order $N_{0}$; those with a single superscript, of order $1 / N_{0}$; those with both (e.g., $M_{I}^{J}$ ) or none (e.g., $P(I)$ ), of $O(1)$; etc. Exceptions are noted with a caret (or "hat"). For example, we denote the "normalized" covariance matrix for the populations (i.e., $\left.\left(\left\langle n_{I} n_{J}\right\rangle-\left\langle n_{I}\right\rangle\left\langle n_{J}\right\rangle\right) / N_{0}\right)$ by $\hat{G}_{I J}$, which is a quantity of $O(1)$ rather than $O\left(N_{0}^{2}\right)$. Similarly, its inverse $\left(\hat{\Gamma}^{I J}\right)$ is also of $O(1)$, as opposed to of $O\left(1 / N_{0}^{2}\right)$. To avoid confusion, we will remind the readers of such exceptions at the appropriate points.

The last ingredient in our model is mutation. In the absence of mutations, the diversity of the population never increases with time. Indeed, the only rigorously stationary state is the collapsed one, $n_{I}=0$ for all $I$. Nevertheless, non-trivial states (with extremely long life times and called TSS's here) are typically reached, for all relevant time scales. While the rigorously stationary state is independent of the intitial condition $\vec{n}(0)$, the TSS states are entirely dependent on the initial population, and they display the dominant characteristics of the corresponding QSS's. Identified by a fixed point (FP) of the deterministic evolution equation,

$$
\left.n_{I}(t+1)=n_{I}(t) F P(I ; \vec{n}(t)\}\right),
$$

a TSS provides the basis for linear stability analysis and for future investigations of the associated QSS.

Returning to mutations, they are important, not only to promote diversity and to model "speciation," but also to provide the main ingredient for the interesting phenomenon of, say, intermittency ("punctuated equilibria") [4]. As in all bit-string models involving asexual reproduction, we allow each offspring to carry the genes of its parent, except for a probability of $\mu / L$ that each bit be changed. As a result, on the average and for small $\mu$, a survivor produces $\mu F$ offspring with different genetic material. To keep track of the "biodiversity," we define the species richness $\mathcal{N}(t)$ as the number of populated species at $t$ (i.e., only $\mathcal{N} I$ 's are present at $t$ ). Another common measure which characterizes the relative abundance of the species better (but will not be the focus here) is the Shannon-Wiener index, $\sum_{J} \rho(J) \ln \rho(J)$, where

$$
\rho(J) \equiv n_{J}(t) / N_{\text {tot }}(t)
$$

is just the fraction of species $J$ in the system.

Let us briefly summarize the algorithm used to simulate this model, referring to [4] for the details. There are three layers of nested loops: (1) over generations $t,(2)$ over $\mathcal{N}(t)$, and (3) over $n_{I}(t)$. In the innermost (last) loop, each individual produces, 
with probability $P(I), F$ offspring, each of which is allowed to mutate before $\vec{n}(t+1)$ is recorded. In most of our studies, we chose $L=13, N_{0}=2000, F=4, \mu=10^{-3}$, and $N_{\text {tot }}(0)=100$, with random initial $\vec{n}(0)$. Thus, $\mathcal{N}_{\max }=8192$, though far fewer species are typically present in the system at any given time, i.e., $\mathcal{N} \ll \mathcal{N}_{\max }$.

\section{Master equation and mean-field theory}

In our recent investigations, the Monte Carlo studies generate stochastic sequences of configurations (i.e., non-negative integers, or just "points," in the $\mathcal{N}_{\text {max }}$-dimensional space) but the theoretical analysis was based on deterministic, heuristic equations of motion for the averages of the populations ("mean-field" theory). To understand the full stochastic process, we need a complete description, involving the probability that the system is found with a specific number of individuals at time $t$, namely, $\mathcal{P}(\vec{n}, t)$. Its evolution is governed by the master equation, given in an appendix of [4. Before turning to this equation, let us emphasize the difference between $\mathcal{P}(\vec{n}, t)$ here and the $\vec{n}(t)$ above. In the former, $\vec{n}$ is a co-ordinate (in $\mathcal{N}_{\text {max }}$-space) and $\mathcal{P}$ an evolving function in this space. By contrast, $\vec{n}(t)$ is just the trajectory of a single point in this space. A single Monte Carlo run generates a particular trajectory (or "history"): $n_{I}(t)$, and can be represented as a (Kronecker) delta function jumping from point to point: $\mathcal{P}_{\text {a MC run }}(\vec{n}, t)=\prod_{I} \delta\left(n_{I}, n_{I}(t)\right)$. The full dynamics of $\mathcal{P}(\vec{n}, t)$ is simulated by averaging over many runs, and thus difficult to access. When we turn out attention to the TSS's below, the process simplifies, since they are characterized by static distributions: $\mathcal{P}^{*}(\vec{n})$. Then, it is sufficient to perform a single, long run during which the system rarely wanders far (say, $O\left(\sqrt{N_{0}}\right)$ ) from the neighborhood of a specific point (typically, fixed points of the mean-field evolution equations).

Returning to the issue at hand, finding an equation for $\mathcal{P}(\vec{n}, t)$, we recapitulate the probability for an individual of species $I$ to survive:

$$
P(I)=\left\{1+\exp \left[\frac{N_{\text {tot }}}{N_{0}}-M_{I}^{J} \frac{n_{J}}{N_{\text {tot }}}\right]\right\}^{-1} .
$$

Again, note the different interpretation we give for this expression versus equation (2.3). Here, $P(I)=P(I ; \vec{n})$ denote $\mathcal{N}_{\text {max }}$ functions defined in $\mathcal{N}_{\text {max }}$-space, independent of $t$. In contrast, for a particular MC run at a particular time $t$, we need only $\mathcal{N}(t)$ functions (for a specific point $\left\{n_{J}(t)\right\}$, in a much smaller, $\mathcal{N}$-space). Next, we must keep track of all of the possible number of survivors. Since all of these individuals reproduce, we will

call them "parents." Defining the symbol $\left[\begin{array}{c}n_{I} \\ m_{I}\end{array}\right]$ as the rate for $m_{I}$ individuals to survive from the original $n_{I}$, we simply write a binomial distribution:

$$
\left[\begin{array}{c}
n_{I} \\
m_{I}
\end{array}\right]=\frac{n_{I} !}{m_{I} !\left(n_{I}-m_{I}\right) !}[P(I)]^{m_{I}}[1-P(I)]^{n_{I}-m_{I}} .
$$

Next, each parent gives rise to $F$ offspring, not every one of which is of the same species. In the simulations, it is possible to have a mutant whose genome differs from the parent by two or more bits. However, this is quite rare, being less than 
$O\left(\mu^{2} N_{\text {tot }}\right)=O\left(\mu^{2} N_{0}\right) \sim 10^{-3}$. Thus, we will keep our analysis simple by restricting our analysis here to mutations which flip only a single bit. Then, there can be only $L+1$ possible varieties of offspring for each parent. To account for these, let us introduce the notation

$b_{J, 0} \quad$ for the number of offspring from parent $J$ with no mutations

$b_{J, \alpha}$ for the number of offspring from parent $J$ with the $\alpha$ th bit flipped

and define the multinomial-like symbol

$$
\left[\begin{array}{c}
F m_{J} \\
b_{J, 0}, b_{J, 1}, \ldots, b_{J, L}
\end{array}\right]=\frac{\left(F m_{J}\right) !}{\left(b_{J, 0}\right) !}(1-\mu)^{b_{J, 0}} \prod_{\alpha=1}^{L} \frac{1}{\left(b_{J, \alpha}\right) !}\left(\frac{\mu}{L}\right)^{b_{J, \alpha}} .
$$

This is the probability that the $F m_{J}$ offspring are distributed into the specific set $\left\{b_{J, 0}, b_{J, 1}, \ldots, b_{J, L}\right\}$. The last ingredient needed is the connection matrix

$$
\Delta_{K}^{J, \alpha}=\left\{\begin{array}{cc}
1 & \text { if genotype } K \text { is } J \text { with the } \alpha \text { th bit flipped } \\
0 & \text { otherwise }
\end{array}\right.
$$

so that the number of offspring born into species $K$ due to mutations is

$$
B_{K} \equiv \sum_{J, \alpha>0} \Delta_{K}^{J, \alpha} b_{J, \alpha}
$$

With these ingredients, we arrive at the master equation $\ddagger$ :

$\mathcal{P}\left(\vec{n}^{\prime}, t+1\right)=\sum_{\vec{n}, \vec{m},\{b\}} \prod_{K} \delta\left(n_{K}^{\prime}, b_{K, 0}+B_{K}\right) \prod_{J}\left[\begin{array}{c}F m_{J} \\ b_{J, 0}, b_{J, 1}, \ldots, b_{J, L}\end{array}\right] \prod_{I}\left[\begin{array}{c}n_{I} \\ m_{I}\end{array}\right] \mathcal{P}(\vec{n}, t)$,

where $\delta\left(n^{\prime}, n\right)$ is the Kronecker delta. Given a particular initial configuration $\vec{n}_{0}, \mathcal{P}(\vec{n}, t)$ can be found, in principle, by recursion with $\mathcal{P}(\vec{n}, 0)=\delta\left(\vec{n}, \vec{n}_{0}\right)$. In that sense, we note that the more precise notation is $\mathcal{P}\left(\vec{n}, t \mid \vec{n}_{0}, 0\right)$, explicitly showing that it is the probability to find our system in state $\vec{n}$ at time $t$, conditioned on a specific initial condition. However, this notation seems unnecessarily cumbersome, so that we will just use $\mathcal{P}(\vec{n}, t)$ in its place.

Note that equation (3.6) is just a special example of the general evolution of conditional probabilities in a Markov process, i.e.,

$$
\mathcal{P}\left(\vec{n}^{\prime}, t+1\right)=\sum_{\vec{n}} R\left(\vec{n}^{\prime} \mid \vec{n}\right) \mathcal{P}(\vec{n}, t)
$$

where $R\left(\vec{n}^{\prime} \mid \vec{n}\right)$ is the conditional probability for finding the system in $\vec{n}^{\prime}$ given that it was in $\vec{n}$, also known as the transition rate. For our case, $R$ is explicitly

$$
R\left(\vec{n}^{\prime} \mid \vec{n}\right)=\sum_{\vec{m},\{b\}} \prod_{K} \delta\left(n_{K}^{\prime}, b_{K}+B_{K}\right) \prod_{J}\left[\begin{array}{c}
F m_{J} \\
b_{J, 0}, b_{J, 1}, \ldots, b_{J, L}
\end{array}\right] \prod_{I}\left[\begin{array}{c}
n_{I} \\
m_{I}
\end{array}\right] .
$$

$\ddagger$ Many master equations are written for continuous time, in the form of $\partial_{t} \mathcal{P}(C, t)=$ $\sum_{C^{\prime}} L\left(C, C^{\prime}\right) \mathcal{P}\left(C^{\prime}, t\right)$. For discrete-time processes, we find it more convenient to express the evolution in the form used here. 
Once $\mathcal{P}(\vec{n}, t)$ is found, the time dependence of the expectation value of any quantity can be obtained via

$$
\langle\bullet\rangle_{t} \equiv \sum_{\vec{n}} \bullet \mathcal{P}(\vec{n}, t)
$$

in principle. Specifically, our main interest here will be $\left\langle n_{I}\right\rangle_{t}$, the average number of individuals of species $I$ at time $t$, as well as quantities quadratic in $n$ (e.g., covariances and correlations). For example, to see how $\left\langle n_{K}\right\rangle_{t}$ evolves in time, we multiply equation (3.7) by $n_{K}^{\prime}$, and sum over $\vec{n}^{\prime}$

$$
\left\langle n_{K}\right\rangle_{t+1}=\left\langle f_{K}(\vec{n})\right\rangle_{t}
$$

where

$$
f_{K}(\vec{n}) \equiv \sum_{\vec{n}^{\prime}} n_{K}^{\prime} R\left(\vec{n}^{\prime} \mid \vec{n}\right)
$$

Exploiting the explicit form of $R$ above and

$$
\sum_{m} m \frac{n !}{m !(n-m) !} q^{m}(1-q)^{n-m}=m q,
$$

it is straightforward to find $f$. Reminding the readers of the $\vec{n}$ dependence in $P$, we write explicitly

$$
f_{K}(\vec{n})=F\left[(1-\mu) n_{K} P(K ; \vec{n})+(\mu / L) \sum_{J} \sum_{\alpha=1}^{L} \Delta_{K}^{J, \alpha} n_{J} P(J ; \vec{n})\right]
$$

The interpretation of various terms in this expression is clear: $F$ offspring are born to $n_{J} P(J)$ survivors of species $J$, with rearrangements into the new generations due to mutations. Inserting it into equation (3.10), we arrive at an exact equation

$$
\left\langle n_{K}\right\rangle_{t+1}=F\left[(1-\mu)\left\langle n_{K} P(K)\right\rangle_{t}+(\mu / L) \sum_{J} \sum_{\alpha=1}^{L} \Delta_{K}^{J, \alpha}\left\langle n_{J} P(J)\right\rangle_{t}\right] .
$$

Its simplicity is deceptive, since the $P$ 's are, from equation (3.1), non-trivial functions of $\vec{n}$. If a formal expansion in powers of $\vec{n}$ were inserted for these functions, then averages of all powers, $\left\langle n_{J} n_{K} \ldots\right\rangle_{t}$, will appear on the right-hand side. Of course, equations for these new averages can be written formally, but the result would be more complex than the BBGKY hierarchy [14]. Needless to say, good approximation schemes are crucial for further progress.

One such scheme, also known as the "mean-field" approximation, is to ignore all correlations in order to produce a closed equation for $\left\langle n_{K}\right\rangle$. Thus, we replace all averages of the products by products of the averages:

$$
\left\langle n_{J} n_{K} \ldots\right\rangle \rightarrow\left\langle n_{J}\right\rangle\left\langle n_{K}\right\rangle \ldots
$$

so that, e.g.,

$$
\left\langle n_{K} P(K ; \vec{n})\right\rangle \rightarrow\left\langle n_{K}\right\rangle P(K ;\langle\vec{n}\rangle) .
$$


In terms of the less cumbersome notation $n_{K}(t) \equiv\left\langle n_{K}\right\rangle_{t}$, equation (3.10) reduces to

$$
n_{K}(t+1)=f_{K}\left(\left\{n_{J}(t)\right\}\right) .
$$

Apart from the change of notation $\left(P_{K}\left(\left\{n_{J}(t)\right\}\right) \rightarrow P(K ; \vec{n}(t))\right)$, this equation is precisely the starting point of our earlier analysis, equation (2) in [4]:

$n_{K}(t+1)=n_{K}(t) F P_{K}\left(\left\{n_{J}(t)\right\}\right)[1-\mu]+(\mu / L) F \sum_{I(K)} n_{I(K)}(t) P_{I(K)}\left(\left\{n_{J}(t)\right\}\right)$,

where $I(K)$ runs over all values of $I$ that differ from $K$ by one bit.

In the low-mutation-rate $(\mu \ll 1)$ regime, much of the behaviour of a QSS is well approximated by a TSS $(\mu=0)$, which can be understood in terms of the fixed points of the mutationless version of this mean-field theory. Here, let us briefly summarize the predictions of this theory. With $\mu=0$, the number of populated species in the system, $\mathcal{N}$, never increases. Therefore, we can restrict our attention to a small subspace $(\mathcal{N}$ dimensional, with $\mathcal{N}<5$ in typical simulation runs) of the full $2^{L}$-dimensional space. In our previous work [4, we use tildes (e.g., $\tilde{\mathbf{M}}$ ) to emphasize this aspect. To keep the notation simple, we will drop the tildes here and keep in mind that, for example, $\mathbf{M}$ is an $\mathcal{N} \times \mathcal{N}$ matrix. Also, with $\mathcal{N}$ being an $O(1)$ quantity (i.e., small compared to $N_{0}$ ), the population of every species is generically $O\left(N_{0}\right)$.

Next, $f_{I}$ simplifies to

$$
f_{I}(\vec{n}) \underset{\mu=0}{\rightarrow} F n_{I} P(I ; \vec{n}) .
$$

Since the fixed point, $\vec{n}^{*}$, obeys

$$
\vec{n}^{*}=\vec{f}\left(\vec{n}^{*}\right),
$$

we have $F P\left(I ; \vec{n}^{*}\right)=1$ for all $I$. Defining the inverse of $\mathbf{M}$ by

$$
\mathbf{W} \equiv \mathbf{M}^{-1}
$$

(with elements $W_{I}^{J}$ ) and the sum

$$
\sigma \equiv \sum_{I J} W_{I}^{J}
$$

we found that the fractions of each species are given by

$$
\rho^{*}(I) \equiv n_{I}^{*} / N_{\text {tot }}^{*}=\sum_{J} W_{I}^{J} / \sigma,
$$

and the total $N_{\text {tot }}^{*}$, by

$$
N_{\text {tot }}^{*} / N_{0}=\ln (F-1)+1 / \sigma .
$$

Finally, the elements of $\mathbf{S}$, the stability matrix, is

$$
S_{I}^{J} \equiv f_{I}^{J}\left(\vec{n}^{*}\right) \equiv \partial f_{I} /\left.\partial n_{J}\right|_{\vec{n}^{*}}=\delta_{I}^{J}+\Lambda_{I}^{J},
$$

where $\delta_{I}^{J}$ is the unit matrix. Here,

$$
\Lambda_{I}^{J}=\left(1-\frac{1}{F}\right)\left(M_{I}^{J}-\ln (F-1)-2 / \sigma\right) \rho^{*}(I) .
$$

(denoted by $\tilde{\Lambda}_{I J}$ in [4] and referred to as the community matrix in biological literature) plays the role of a "restoring" force, driving the population back towards the (stable) fixed point. 


\section{Fluctuations and correlations of populations}

It is clear that all stochastic aspects of the system are lost in the mean-field approximation. In this section, we study the simplest aspect, namely, an approximate description of the long-lived, quasi-steady states (QSS). Now, as we have seen in simulations [4], these states are dominated by a few mutually supportive species, along with a few individuals of closely related "benign mutants." Thus, we restrict ourselves in this paper to systems without mutation, so that several simplifications apply. First, there is the obvious reduction of $R$ from equation (3.8) to

$$
R\left(\vec{n}^{\prime} \mid \vec{n}\right) \underset{\mu=0}{\rightarrow} \sum_{\vec{m}} \prod_{K} \delta\left(n_{K}^{\prime}, F m_{K}\right) \prod_{I}\left[\begin{array}{c}
n_{I} \\
m_{I}
\end{array}\right] .
$$

Next, since there can be no new species, we can focus on populations with $\mathcal{N} \sim O(1)$ species, each of which having $O\left(N_{0}\right)$ individuals. Finally, though it is possible for a fluctuation to collapse the entire population (which is, rigorously, the unique stationary state associated with equations (3.74.10), such events are so extreme that the life times of a typical non-trivial state should be $O\left(\mathrm{e}^{N_{0}}\right)$. So, for all practical purposes, we may regard such states as "truly" stationary (the TSS's). $\S$

A complete description of a TSS is provided by a stationary distribution $\mathcal{P}^{*}(\vec{n})$, which satisfies

$$
\mathcal{P}^{*}\left(\vec{n}^{\prime}\right)=\sum_{\vec{n}} R\left(\vec{n}^{\prime} \mid \vec{n}\right) \mathcal{P}^{*}(\vec{n}) .
$$

Given $\mathcal{P}^{*}(\vec{n})$, we can find stationary averages of any quantity:

$$
\langle\bullet\rangle^{*} \equiv \sum_{\vec{n}}(\bullet) \mathcal{P}^{*}(\vec{n})
$$

Here, our main interest will be mean populations and their correlations:

$$
\left\langle n_{I}\right\rangle^{*} \text { and }\left\langle n_{I} n_{J}\right\rangle^{*}-\left\langle n_{I}\right\rangle^{*}\left\langle n_{J}\right\rangle^{*} \text {. }
$$

Even in the absence of mutations and with simplified $R$ 's, there are substantial non-linearities (through $P(\vec{n})$ ) in the problem that prevent us from finding solutions to equation (4.2) in general. On the other hand, observations in simulations (and lessons learned from the central limit theorem) show that our distributions are well approximated by Gaussians. Indeed, a systematic expansion for $\mathcal{P}^{*}$ can be formulated, starting from the Gaussian form:

$\mathcal{P}_{\mathrm{G}}(\vec{n})=\left(2 \pi N_{0}\right)^{-\mathcal{N} / 2}(\operatorname{det} \hat{\Gamma})^{1 / 2} \exp \left[-\frac{1}{2 N_{0}}\left(n_{I}-\bar{n}_{I}\right) \hat{\Gamma}^{I J}\left(n_{J}-\bar{n}_{J}\right)\right]$.

where $\bar{n}_{I}$ and $\hat{\Gamma}^{I J}$ are parameters to be determined. Of course, the first of these is of $O\left(N_{0}\right)$. As for the latter, we expect the fluctuations in our problem to be $O\left(\sqrt{N_{0}}\right)$,

$\S$ It is possible to force such states to be rigorously stationary, by slight modifications of the rates. The simplest is to let the survival probabilities $P(I)$ be unity when $n_{I}=1$. 
i.e., covariances of $O\left(N_{0}\right)$. To incorporate this expectation, we have chosen to write $\mathcal{P}_{\mathrm{G}}$ in a form such that the matrix $\hat{\Gamma}$ has elements of $O(1)$ :

$$
\hat{\Gamma}^{I J} \sim O(1)
$$

despite the presence of two superscripts.

Within the context of such a scheme, it is possible to compute these quantities from the microscopic rates. Before proceeding to this computation, we remark that this approximation for $\mathcal{P}^{*}$ will lead to the predictions

$$
\left\langle n_{I}\right\rangle^{*}=\bar{n}_{I} \quad \text { and } \quad\left\langle n_{I} n_{J}\right\rangle^{*}-\left\langle n_{I}\right\rangle^{*}\left\langle n_{J}\right\rangle^{*}=N_{0} \hat{G}_{I J},
$$

where $\hat{\mathbf{G}}$ is the inverse of $\hat{\boldsymbol{\Gamma}}$, i.e.,

$$
\hat{G}_{I J} \hat{\Gamma}^{J K}=\delta_{I}^{K} .
$$

Of course, we expect

$$
\hat{G}_{I J} \sim O(1)
$$

despite its two subscripts.

Though such approaches are well known [15], we provide a few details in the Appendix, both for the sake of completeness and for the convenience of readers unfamiliar with these methods. Here, we present only the highlights of the analysis and how they apply to our case. As shown below, the agreement between our predictions and simulation data in three specific cases are excellent and validates this entire approach.

Turning to the computation of $\bar{n}_{I}$ and $\hat{\boldsymbol{\Gamma}}$ (or $\hat{\mathbf{G}}$ ), we may expect the former to be simply related to the $n_{I}^{*}$ of mean-field theory (equation (3.20)). As shown in the appendix, we have

$$
\bar{n}_{I}=n_{I}^{*}\left[1+O\left(1 / N_{0}\right)\right]
$$

(provided none of the eigenvalues of $\mathbf{S}$ are close to unity). Indeed, the first correction can be computed explicitly. Since these corrections are not necessary for finding the fluctuations and correlations, we will not quote the results here, but refer the reader to equation (A.22) in the Appendix. To find $\hat{\mathbf{G}}$, we need not only $f_{I}(\vec{n})$ and the stability matrix $\mathbf{S}$ (given by equations (3.1913.2513.26)), but also

$$
H_{I J}(\vec{n}) \equiv \sum_{\vec{n}^{\prime}} n_{I}^{\prime} n_{J}^{\prime} R\left(\vec{n}^{\prime} \mid \vec{n}\right)
$$

In the limit $\mu \rightarrow 0, R$ reduces to equation (4.1), so that

$$
\begin{aligned}
H_{I J}(\vec{n}) & \rightarrow \sum_{\vec{m}}\left(F m_{I}\right)\left(F m_{J}\right) \prod_{I}\left[\begin{array}{c}
n_{I} \\
m_{I}
\end{array}\right] \\
& =F^{2}\left[n_{I} n_{J} P(I) P(J)+\delta_{I}^{J} n_{I} P(I)(1-P(I))\right],
\end{aligned}
$$

which is indeed of the form in equation (A.26): $H_{I J}=f_{I} f_{J}+N_{0} \hat{H}_{I J}$. Thus, we readily identify $\hat{H}_{I J}$ of equation (A.30):

$$
\hat{H}_{I J}=\delta_{I}^{J} F^{2} n_{I}^{*} P\left(I ; \vec{n}^{*}\right)\left[1-P\left(I ; \vec{n}^{*}\right)\right] / N_{0} .
$$



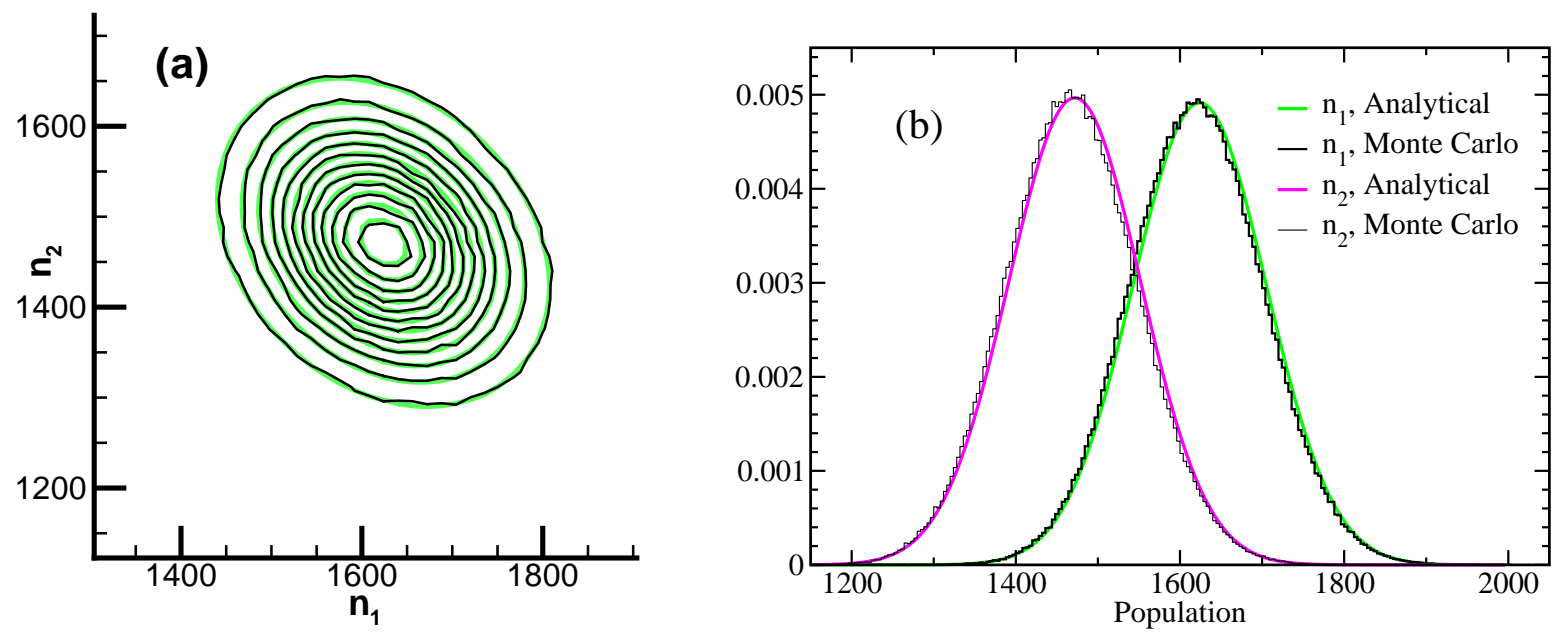

Figure 1. Theoretical Gaussian (thick, grey curves in the background) and simulated (thin, black curves in the foreground) TSS probability densities for $\mathcal{N}=2, F=4$, and $N_{0}=2000$, corresponding to the second column in table 11 The Monte Carlo simulation was performed over 524,290 generations with zero probability of mutations. (a) Contour plot of the joint distribution for $n_{1}$ and $n_{2}$, equation (4.5). The negative slope of the long axis indicates that $n_{1}$ and $n_{2}$ are negatively correlated. (b) Marginal distributions for $n_{1}$ (right) and $n_{2}$ (left), equation (4.22).

But, $F P\left(I ; \vec{n}^{*}\right)=1$ for all $I$, so that

$$
\hat{H}_{I J}=\delta_{I}^{J}(F-1) n_{I}^{*} / N_{0} \text {. }
$$

Referring to the Appendix again for the details, $\hat{\mathbf{G}}$ can be obtained from $\mathbf{S}$ and $\hat{\mathbf{H}}$ via the linear relationship

$$
\hat{\mathbf{G}}-\mathbf{S} \hat{\mathbf{G}} \mathbf{S}^{T}=\hat{\mathbf{H}} \text {. }
$$

Inserting the explicit form for $\hat{\mathbf{H}}$ into equations A.39, A.40), we arrive at a complete solution for the covariance matrix in terms of $\lambda_{a}, u_{a}^{K}$, and $v_{I}^{a}$ (respectively, the eigenvalues and the left and right eigenvectors of $S_{K}^{I}$, normalized by $\left.u_{a}^{I} v_{I}^{b}=\delta_{a}^{b}\right)$ :

$$
\hat{G}_{I J}=(F-1) \sum_{a, b, K} v_{I}^{a} v_{J}^{b} u_{a}^{K} u_{b}^{K} \frac{n_{K}^{*} / N_{0}}{1-\lambda_{a} \lambda_{b}}+O\left(1 / N_{0}\right) .
$$

Before comparing these predictions to simulations, we provide an intuitive picture for these fluctuations and correlations. Since we are concerned with distributions well approximated by Gaussians, the underlying process is just an Ornstein-Uhlenbeck one 15. Such a process can be described by a Langevin equation:

$$
\vec{\varepsilon}(t+1)-\vec{\varepsilon}(t)=\Lambda \vec{\varepsilon}(t)+\vec{\eta}(t),
$$

where $\boldsymbol{\Lambda}$ plays the role of restoring forces which drive $\vec{\varepsilon}$ towards zero, and $\vec{\eta}$ is a Gaussian noise with

$$
\langle\vec{\eta}(t)\rangle=0 ;\left\langle\vec{\eta}(t) \vec{\eta}\left(t^{\prime}\right)\right\rangle=\hat{\mathbf{H}} \delta\left(t, t^{\prime}\right)
$$


The notation for the two matrices $(\boldsymbol{\Lambda}, \hat{\mathbf{H}})$ is chosen deliberately. For this problem, they are precisely those given above: equations (3.264.14), while $\vec{\varepsilon}(t)$ is just the deviation from the average:

$$
\vec{\varepsilon}(t)=\vec{n}(t)-\bar{n} .
$$

The deterministic part of equation (4.18) is intuitively clear, being the same as in the mean-field evolution equation, linearized about the fixed point. Now, $\vec{\eta}$ can be seen as the (microscopic) noise in the stochastic process. In our case, this is clearly due to the uncertainties associated with survival. Since we have a simple two-state (dying or surviving) random process, we can hardly be surprised by the presence of the factors $n P(1-P)$. Also, since this randomness is imposed on each individual, the diagonal form of $\hat{\mathbf{H}}$ can be anticipated. Perhaps only the factor $F^{2}$ cannot be easily surmised.

We have carried out simulations for ten cases, with $\mathcal{N}=2,3$, and 4 . For simplicity, in each case we chose a set of species which served as the dominant ones in one of the ten QSS communities included in Table I of [4. Of course, since $\mu$ was set to zero in the simulations reported here, the communities are TSS's. The runs were carried out for 524,290 generations each. From the recorded $n_{I}(t)$, we computed the averages $\left\langle n_{I}\right\rangle_{\mathrm{MC}}$ and the covariance matrix $\hat{\mathbf{G}}_{\mathrm{MC}}$. For the $\mathcal{N}=2$ case, we can easily display a histogram of all the populations (figure 1(a)). In addition, in figure 1(b), we show how good the Gaussian approximation is by plotting the projections of both this histogram (onto one or the other axis) and the theoretical predictions, e.g.,

$$
\begin{aligned}
\mathcal{P}_{\text {proj }}\left(n_{1}\right) & =\int \mathrm{d} n_{2} \mathcal{P}_{\mathrm{G}}\left(n_{1}, n_{2}\right) \\
& =\left(\frac{\operatorname{det} \hat{\boldsymbol{\Gamma}}}{2 \pi \hat{N}_{0} \hat{\Gamma}_{22}}\right)^{1 / 2} \exp \left[-\frac{\operatorname{det} \hat{\boldsymbol{\Gamma}}}{2 N_{0} \hat{\Gamma}_{22}}\left(n_{1}-\bar{n}_{1}\right)^{2}\right] .
\end{aligned}
$$

We emphasize that the theoretical curves are produced with no fitting parameters all quantities were computed from the model specifications (i.e., $N_{0}, F$, and $\mathbf{M}$ ). For the $\mathcal{N}>2$ cases, it is difficult to display full histograms. Instead, we only provide the comparison for the averages and covariance matrices for three particular TSS's in tables 1 and 2. As we see, the agreement is excellent, well within the expected accuracy of the approximation, $O\left(1 / N_{0}\right)$, and the statistical errors, $O(1 / \sqrt{524,290})$.

\section{Distributions containing dynamical information}

In the previous section, we focused on the static distribution of the populations in a TSS. In other words, we can compute (within the Gaussian approximation) correlation functions that involve any number of species, all "at the same time." Here, we investigate the information contained in the dynamics of the stochastic process, i.e., time-dependent correlations. For an evolving population, a natural question is how the system changes from one generation to the next. To probe this issue at a quantitative level, let us consider two examples, the statistics of "steps,"

$$
\vec{s}(t) \equiv \vec{n}(t+1)-\vec{n}(t),
$$


Table 1. Theoretical results for two TSS communities with $\mathcal{N}=2$ and 3 , respectively, compared with corresponding quantities from Monte Carlo simulations with $N_{0}=2000$ over 524,290 generations. The communities are defined by the interaction matrices M. The quantities shown are the normalized fixed-point population vector, $\vec{n}^{*} / N_{0}$ [equations [3.23) and (3.24)] and the corresponding Monte Carlo average $\langle\vec{n}\rangle_{\mathrm{MC}} / N_{0}$, the normalized population covariance matrix $\hat{\mathbf{G}}$ [defined by equation (4.7) and calculated from equation (A.36) with 20 iterations for $\mathcal{N}=2$ and 30 iterations for $\mathcal{N}=3$ ] and $\hat{\mathbf{G}}_{\mathrm{MC}}$, the step-covariance matrix $\hat{\mathrm{g}}$ [equation (5.17)] and $\hat{\mathrm{g}}_{\mathrm{MC}}$, and the normalized correlation matrix between steps $\vec{s}(t)$ and the deviation from the average population $\vec{\varepsilon}(t)=\vec{n}(t)-\vec{n}^{*}$, $\hat{\mathbf{C}}$ [equation (5.22)] and $\hat{\mathbf{C}}_{\mathrm{MC}}$. All numbers are given to four significant digits. Results for $\mathcal{N}=4$ are shown in table 2

\begin{tabular}{|c|c|c|c|c|c|}
\hline $\mathcal{N}$ & \multicolumn{2}{|c|}{2} & \multicolumn{3}{|c|}{3} \\
\hline $\mathbf{M}$ & $\left(\begin{array}{c}0 \\
0.8563\end{array}\right.$ & $\left.\begin{array}{c}0.9448 \\
0\end{array}\right)$ & $\left(\begin{array}{c}0 \\
0.8935 \\
0.6474\end{array}\right.$ & $\begin{array}{c}0.7497 \\
0 \\
0.9881\end{array}$ & $\left.\begin{array}{c}0.9450 \\
0.6212 \\
0\end{array}\right)$ \\
\hline$\vec{n}^{*} / N_{0}$ & $\left(\begin{array}{l}0.81 \\
0.73\end{array}\right.$ & $\left.\begin{array}{l}19 \\
59\end{array}\right)$ & & $\left.\begin{array}{l}0.6062 \\
0.4897 \\
0.5388\end{array}\right)$ & \\
\hline$\langle\vec{n}\rangle_{\mathrm{MC}} / N_{0}$ & $\left(\begin{array}{l}0.81 \\
0.73\end{array}\right.$ & $\left.\begin{array}{l}12 \\
50\end{array}\right)$ & & $\left.\begin{array}{l}0.6055 \\
0.4892 \\
0.5378\end{array}\right)$ & \\
\hline$\hat{\mathbf{G}}$ & $\begin{array}{c}3.294 \\
-0.8722\end{array}$ & $\left.\begin{array}{c}-0.8722 \\
3.224\end{array}\right)$ & $\left(\begin{array}{c}3.667 \\
-0.9323 \\
-0.9558\end{array}\right.$ & $\begin{array}{c}-0.9323 \\
3.551 \\
-0.9249\end{array}$ & $\begin{array}{c}-0.9558 \\
-0.9249 \\
3.590\end{array}$ \\
\hline$\hat{\mathbf{G}}_{\mathrm{MC}}$ & $\begin{array}{c}3.295 \\
-0.8784\end{array}$ & $\left.\begin{array}{c}-0.8784 \\
3.227\end{array}\right)$ & $\left(\begin{array}{c}3.690 \\
-0.9440 \\
-0.9584\end{array}\right.$ & $\begin{array}{c}-0.9440 \\
3.573 \\
-0.9289\end{array}$ & $\begin{array}{c}-0.9584 \\
-0.9289 \\
3.592\end{array}$ \\
\hline$\hat{\mathrm{g}}$ & $\left(\begin{array}{l}4.455 \\
1.368\end{array}\right.$ & $\left.\begin{array}{l}1.368 \\
3.882\end{array}\right)$ & $\left(\begin{array}{c}3.039 \\
0.7902 \\
0.8768\end{array}\right.$ & $\begin{array}{c}0.7902 \\
2.285 \\
0.7152\end{array}$ & $\left.\begin{array}{c}0.8768 \\
0.7152 \\
2.592\end{array}\right)$ \\
\hline$\hat{\mathrm{g}}_{\mathrm{MC}}$ & $\left(\begin{array}{l}4.442 \\
1.352\end{array}\right.$ & $\left.\begin{array}{l}1.352 \\
3.865\end{array}\right)$ & $\left(\begin{array}{c}3.050 \\
0.7865 \\
0.8716\end{array}\right.$ & $\begin{array}{c}0.7865 \\
2.287 \\
0.7098\end{array}$ & $\left.\begin{array}{c}0.8716 \\
0.7098 \\
2.586\end{array}\right)$ \\
\hline$\hat{\mathrm{C}}$ & $\begin{array}{c}-2.227 \\
-0.7189\end{array}$ & $\left.\begin{array}{c}-0.6494 \\
-1.941\end{array}\right)$ & $\left(\begin{array}{c}-1.520 \\
-0.2649 \\
-0.5956\end{array}\right.$ & $\begin{array}{c}-0.5253 \\
-1.143 \\
-0.1908\end{array}$ & $\begin{array}{c}-0.2812 \\
-0.5244 \\
-1.296\end{array}$ \\
\hline$\hat{\mathbf{C}}_{\mathrm{MC}}$ & $\begin{array}{c}-2.221 \\
-0.7139\end{array}$ & $\left.\begin{array}{c}-0.6385 \\
-1.933\end{array}\right)$ & $\left(\begin{array}{c}-1.524 \\
-0.2643 \\
-0.5902\end{array}\right.$ & $\begin{array}{c}-0.5221 \\
-1.143 \\
-0.1873\end{array}$ & $\begin{array}{c}-0.2814 \\
-0.5224 \\
-1.293\end{array}$ \\
\hline
\end{tabular}


Table 2. Theoretical results for a TSS community with $\mathcal{N}=4$, compared with corresponding quantities from a Monte Carlo simulation with $N_{0}=10,000$ over 524,290 generations. The quantities shown are the same as in table 1 The eigenvalues of $\mathbf{S}$ are relatively close to unity, so evaluation of $\mathbf{G}$ from equation A.36 required 180 iterations.

\begin{tabular}{|c|c|c|c|c|}
\hline $\mathcal{N}$ & & & 4 & \\
\hline M & $\left(\begin{array}{c}0 \\
0.9543 \\
0.4724 \\
0.9734\end{array}\right.$ & $\begin{array}{cc} & 0.5507 \\
3 & 0 \\
4 & 0.6190 \\
4 & 0.6808\end{array}$ & $\begin{array}{c}0.5101 \\
0.8437 \\
0 \\
0.1862\end{array}$ & $\left.\begin{array}{c}0.9847 \\
0.9508 \\
0.7371 \\
0\end{array}\right)$ \\
\hline$\vec{n}^{*} / N_{0}$ & \multicolumn{4}{|c|}{$\left(\begin{array}{l}0.3355 \\
0.7034 \\
0.2366 \\
0.3468\end{array}\right)$} \\
\hline$\langle\vec{n}\rangle_{\mathrm{MC}} / N_{0}$ & \multicolumn{4}{|c|}{$\left(\begin{array}{l}0.3356 \\
0.7035 \\
0.2354 \\
0.3472\end{array}\right)$} \\
\hline$\hat{\mathbf{G}}$ & $\left(\begin{array}{c}4.215 \\
-1.043 \\
-2.583 \\
1.132\end{array}\right.$ & $\begin{array}{c}-1.043 \\
3.882 \\
-0.1463 \\
-1.006\end{array}$ & $\begin{array}{c}-2.583 \\
-0.1463 \\
6.509 \\
-3.791\end{array}$ & $\left.\begin{array}{c}1.132 \\
-1.006 \\
-3.791 \\
5.653\end{array}\right)$ \\
\hline$\hat{\mathbf{G}}_{\mathrm{MC}}$ & $\left(\begin{array}{c}4.252 \\
-1.055 \\
-2.620 \\
1.151\end{array}\right.$ & $\begin{array}{c}-1.055 \\
3.870 \\
-0.1366 \\
-1.003\end{array}$ & $\begin{array}{c}-2.620 \\
-0.1366 \\
6.612 \\
-3.879\end{array}$ & $\left.\begin{array}{c}1.152 \\
-1.003 \\
-3.879 \\
5.718\end{array}\right)$ \\
\hline$\hat{\mathrm{g}}$ & $\left(\begin{array}{l}1.387 \\
0.6468 \\
0.2384 \\
0.3094\end{array}\right.$ & $\begin{array}{lc} & 0.6468 \\
8 & 3.704 \\
4 & 0.4482 \\
4 & 0.6518\end{array}$ & $\begin{array}{l}0.2384 \\
0.4482 \\
0.8927 \\
0.2414\end{array}$ & $\left.\begin{array}{c}0.3094 \\
0.6518 \\
0.2414 \\
1.461\end{array}\right)$ \\
\hline$\hat{\mathrm{g}}_{\mathrm{MC}}$ & $\left(\begin{array}{l}1.388 \\
0.6417 \\
0.2368 \\
0.3090\end{array}\right.$ & $\begin{array}{cc} & 0.6417 \\
7 & 3.694 \\
8 & 0.4440 \\
0 & 0.6501\end{array}$ & $\begin{array}{l}0.2368 \\
0.4440 \\
0.8870 \\
0.2381\end{array}$ & $\left.\begin{array}{c}0.3090 \\
0.6501 \\
0.2381 \\
1.462\end{array}\right)$ \\
\hline$\hat{\mathbf{C}}$ & $\begin{array}{l}-0.6934 \\
-0.2517 \\
-0.1655 \\
-0.1253\end{array}$ & $\begin{array}{l}-0.3951 \\
-1.852 \\
-0.2681 \\
-0.3237\end{array}$ & $\begin{array}{l}-0.07285 \\
-0.1801 \\
-0.4464 \\
-0.2209\end{array}$ & $\left.\begin{array}{c}-0.1841 \\
-0.3281 \\
-0.02048 \\
-0.7304\end{array}\right)$ \\
\hline$\hat{\mathbf{C}}_{\mathrm{MC}}$ & $\begin{array}{l}-0.6942 \\
-0.2510 \\
-0.1642 \\
-0.1239\end{array}$ & $\begin{array}{l}-0.3907 \\
-1.847 \\
-0.2661 \\
-0.3242\end{array}$ & $\begin{array}{l}-0.07252 \\
-0.1779 \\
-0.4435 \\
-0.2202\end{array}$ & $\left.\begin{array}{c}-0.1851 \\
-0.3259 \\
-0.01783 \\
-0.7310\end{array}\right)$ \\
\hline
\end{tabular}


and the correlation of these steps with the deviations from the average: $\vec{\varepsilon}(t)$.

In a stationary state, the mean-field prediction for the step size is clearly zero $\left(\vec{s}^{\mathrm{MF}} \equiv 0\right)$, as must also be the case for the average $\langle\vec{s}\rangle^{*}$. Nevertheless, we expect a typical step size to be $O\left(\sqrt{N_{0}}\right)$. For a more detailed picture, we may seek the step-size distribution $(\mathrm{SSD})$ in the steady state: $\mathcal{P}_{s}^{*}(\vec{s})$. A precise definition is

$$
\mathcal{P}_{s}^{*}(\vec{s}) \equiv \lim _{t \rightarrow \infty} \sum_{\vec{n}^{\prime}, \vec{n}} \delta\left(\vec{s}, \vec{n}^{\prime}-\vec{n}\right) \mathcal{P}\left(\vec{n}^{\prime}, t+1 ; \vec{n}, t\right),
$$

where $\mathcal{P}\left(\vec{n}^{\prime}, t+1 ; \vec{n}, t\right)$ is the joint probability for finding the system with population $\vec{n}$ at time $t$ and with $\vec{n}^{\prime}$ in the next step. From the master equation (3.7), it is clear that this is just $R\left(\vec{n}^{\prime} \mid \vec{n}\right) \mathcal{P}(\vec{n}, t)$, so that

$$
\lim _{t \rightarrow \infty} \mathcal{P}\left(\vec{n}^{\prime}, t+1 ; \vec{n}, t\right)=R\left(\vec{n}^{\prime} \mid \vec{n}\right) \mathcal{P}^{*}(\vec{n}) .
$$

Thus, once the steady-state distribution $\mathcal{P}^{*}(\vec{n})$ is known, the SSD can be obtained from:

$$
\mathcal{P}_{s}^{*}(\vec{s})=\sum_{\vec{n}} R(\vec{n}+\vec{s} \mid \vec{n}) \mathcal{P}^{*}(\vec{n}) .
$$

Applying this formalism to our case of coevolving species without mutations, we can exploit all the approximations detailed in the previous section, namely, a Gaussian for the stationary state $\left(\mathcal{P}^{*}(\vec{n}) \cong \mathcal{P}_{\mathrm{G}}(\vec{n})\right.$ of equation (4.5)), continuous variables for $\vec{n}$, and integrals instead of sums. Given equation (4.2), the success of that scheme is implicitly dependent on the fact that $R\left(\vec{n}^{\prime} \mid \vec{n}\right)$ is also well approximated by a Gaussian $\|$. As a result, we need not carry out another lengthy analysis to conclude that $\mathcal{P}_{s}^{*}(\vec{s})$ should also be of the form

$$
\mathcal{P}_{s}^{*}(\vec{s}) \cong\left(2 \pi N_{0}\right)^{-\mathcal{N} / 2}(\operatorname{det} \hat{\gamma})^{1 / 2} \exp \left[-\frac{1}{2 N_{0}} s_{I} \hat{\gamma}^{I J} s_{J}\right]
$$

However, it is clear that the matrix $\hat{\gamma}$ is distinct from $\hat{\boldsymbol{\Gamma}}$, since the former must contain some "dynamic" information. (Note that the caret is to remind us that, despite the presence of two superscripts, $\hat{\gamma}^{I J}$ is of $O(1)$.) Now, within the context of simple Gaussians, $\hat{\gamma}$ can be found by computing its inverse

$$
\hat{\mathrm{g}} \equiv \hat{\gamma}^{-1}
$$

which is just the second moment

$$
\left\langle s_{I} s_{J}\right\rangle_{s} \equiv \sum_{\vec{s}} s_{I} s_{J} \mathcal{P}_{s}^{*}(\vec{s})
$$

Let us first derive an exact formula for this quantity in terms of $\langle\bullet\rangle^{*}$. Starting with

$$
\begin{aligned}
\left\langle s_{I} s_{J}\right\rangle_{s} & =\sum_{\vec{s}, \vec{n}} s_{I} s_{J} R(\vec{n}+\vec{s} \mid \vec{n}) \mathcal{P}^{*}(\vec{n}) \\
& =\sum_{\vec{n}^{\prime}, \vec{n}}\left(n_{I}^{\prime}-n_{I}\right)\left(n_{J}^{\prime}-n_{J}\right) R\left(\vec{n}^{\prime} \mid \vec{n}\right) \mathcal{P}^{*}(\vec{n}),
\end{aligned}
$$

\|In the same manner as for the stationary distributions, this property can be derived from the definition of $R$ using straightforward, but tedious, manipulations with the binomials. Here, we can treat this as an assumption, the justification of which will be the agreement with simulation data. 
we use equations (4.2) and (3.11) and arrive at the exact relation:

$$
\left\langle s_{I} s_{J}\right\rangle_{s}=2\left\langle n_{I} n_{J}\right\rangle^{*}-\left\langle f_{I} n_{J}\right\rangle^{*}-\left\langle n_{I} f_{J}\right\rangle^{*} .
$$

Next, we exploit $\mathcal{P}^{*}(\vec{n}) \cong \mathcal{P}_{\mathrm{G}}(\vec{n})$ for computing $\langle\bullet\rangle^{*}$ and apply equation (A.17). Thus,

$$
\left\langle n_{I} n_{J}\right\rangle^{*} \cong \bar{n}_{I} \bar{n}_{J}+N_{0} \hat{G}_{I J}
$$

and

$$
\left\langle f_{I} n_{J}\right\rangle^{*} \cong f_{I}(\bar{n}) \bar{n}_{J}+N_{0} f_{I}^{K}(\bar{n}) \hat{G}_{K J}+\frac{N_{0}}{2} f_{I}^{K M}(\bar{n}) \bar{n}_{J} \hat{G}_{K M} .
$$

Thanks to equation (A.18), the first and the last terms in equation (5.12) combine, so that

$$
\left\langle f_{I} n_{J}\right\rangle^{*} \cong \bar{n}_{I} \bar{n}_{J}+N_{0} f_{I}^{K}(\bar{n}) \hat{G}_{K J} .
$$

To the order kept here, $f_{I}^{K}(\bar{n})$ is just $f_{I}^{K}\left(\vec{n}^{*}\right)=S_{I}^{K}$. So, collecting various items and using equation (3.25), we arrive at

$$
\begin{aligned}
\left\langle s_{I} s_{J}\right\rangle_{s} & \cong N_{0}\left(2 \hat{G}_{I J}-S_{I}^{K} \hat{G}_{K J}-\hat{G}_{I K} S_{J}^{K}\right) \\
& =-N_{0}\left(\Lambda_{I}^{K} \hat{G}_{K J}+\hat{G}_{I K} \Lambda_{J}^{K}\right) .
\end{aligned}
$$

Finally, we relate this result to the Gaussian approximation equation (5.5) intended for the SSD, which provides

$$
\left\langle s_{I} s_{J}\right\rangle_{s} \cong N_{0} \hat{g}_{I J}
$$

and obtain a simple equation for $\hat{\mathrm{g}}$ :

$$
\hat{\mathrm{g}}=-\left(\Lambda \hat{\mathrm{G}}+\hat{\mathbf{G}} \boldsymbol{\Lambda}^{T}\right)
$$

As in the previous section, these predictions are well borne out in simulations. For the same $\mathcal{N}=2$ case as above, we display a two-dimensional histogram of the step sizes in figure 2. Note that this distribution is indeed quite different from that for the populations (figure 1). For the $\mathcal{N}>2$ cases, full histograms are difficult to display and we only show the correlation matrices $\hat{\mathrm{g}}$ and their Monte Carlo counterparts $\hat{\mathrm{g}}_{\mathrm{MC}}$ in tables 1 and 2

Although the SSD probes the underlying dynamics, it does not contain all information of the stochastic (Ornstein-Uhlenbeck) process. In particular, equation (5.17) shows that the antisymmetric part of $\Lambda \hat{\mathbf{G}}$ is "missing." To remedy this shortcoming, we turn to another question which naturally comes to mind: How are the steps $(\vec{s})$ correlated with the deviations from the average $(\vec{\varepsilon} \equiv \vec{n}-\bar{n})$ ? Note that, since two quantities are involved $(\vec{s}$ and $\vec{\varepsilon})$, a full distribution associated with this question is slightly more involved than $\mathcal{P}^{*}(\vec{n})$ or $\mathcal{P}_{s}^{*}(\vec{s})$. Nevertheless, within the approximation scheme we use, it would be a (generalized) Gaussian in the steady state. For simplicity, let us focus on, instead of the full distribution, only the normalized correlation of $\vec{s}$ with $\vec{\varepsilon}:$

$$
\hat{C}_{I J} \equiv\left\langle s_{I} \varepsilon_{J}\right\rangle / N_{0}
$$




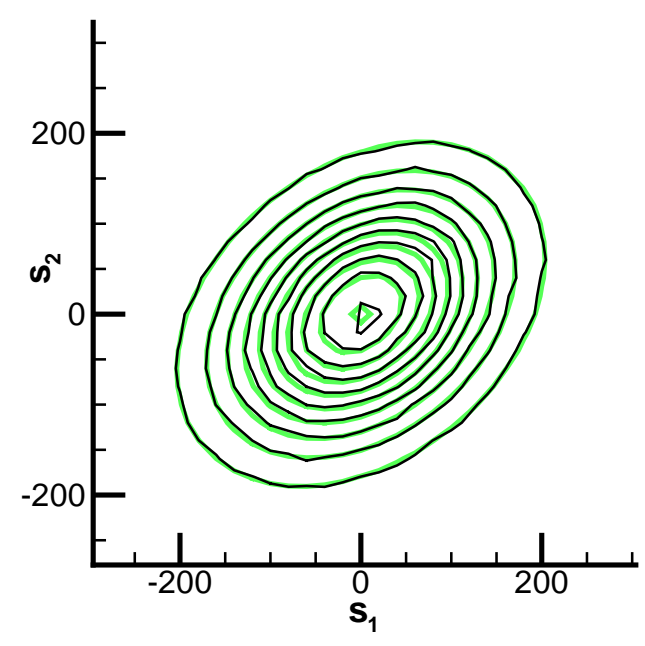

Figure 2. Contour plot of the theoretical Gaussian and simulated joint probability densities for the steps, $s_{1}$ and $s_{2}$, equation (5.5). The line types and parameters are the same as in figure 1 and the simulated histogram was obtained from the same simulation run as in that figure. The positive slope of the long axis indicates that the steps are positively correlated.

By using this notation, we are displaying again our expectation that $\left\langle s_{I} \varepsilon_{J}\right\rangle$ is also of $O\left(N_{0}\right)$. Starting at the same point as equation (5.8), we write

$$
\begin{aligned}
\left\langle s_{I} \varepsilon_{J}\right\rangle & =\sum_{\vec{s}, \vec{n}} s_{I} \varepsilon_{J} R(\vec{n}+\vec{s} \mid \vec{n}) \mathcal{P}^{*}(\vec{n}) \\
& =\sum_{\vec{n}^{\prime}, \vec{n}}\left(n_{I}^{\prime}-n_{I}\right)\left(n_{J}-\bar{n}_{J}\right) R\left(\vec{n}^{\prime} \mid \vec{n}\right) \mathcal{P}^{*}(\vec{n}) .
\end{aligned}
$$

Since $\left\langle\vec{n}^{\prime}\right\rangle^{*}=\langle\vec{n}\rangle^{*}$, this reduces to

$$
\left\langle s_{I} \varepsilon_{J}\right\rangle=\left\langle f_{I} n_{J}\right\rangle^{*}-\left\langle n_{I} n_{J}\right\rangle^{*},
$$

which is again an exact relationship. Repeating the analysis in the previous paragraph, we find, in the Gaussian approximation, a simple result: $\left\langle s_{I} \varepsilon_{J}\right\rangle=N_{0} \Lambda_{I}^{K} \hat{G}_{K J}$, or

$$
\hat{\mathbf{C}}=\Lambda \hat{\mathbf{G}} \text {. }
$$

Unlike the step-size covariance, this matrix is not symmetric in general and contains the full information of the dynamics (to this order of our approximation). Note also that, since $\boldsymbol{\Lambda}$ is negative and $\hat{\mathbf{G}}$ is positive, this correlation is typically negative. This simply reflects a restoring dynamics: steps and deviations from the mean tend to be opposite. In tables 1 and 2, we see that there is excellent agreement between the predicted $\hat{\mathbf{C}}$ 's and their counterparts from simulations.

Another commonly studied correlation is the connected two-time function, $\left(\left\langle n_{I}(t+\tau) n_{J}(t)\right\rangle-\left\langle n_{I}\right\rangle\left\langle n_{J}\right\rangle\right) / N_{0}$, which, in the stationary state, is just

$$
\hat{\Xi}_{I J}(\tau)=\left\langle\varepsilon_{I}(t+\tau) \varepsilon_{I}(t)\right\rangle / N_{0} \text {. }
$$


Within the Gaussian approximation, equation (4.18) can be used repeatedly to express $\varepsilon(t+\tau)$ in terms of $\varepsilon(t)$ and the noise, $\eta_{I}\left(t+t^{\prime}\right)$ at each time step. Since $\left\langle\eta_{I}\left(t+t^{\prime}\right) \varepsilon(t)\right\rangle=$ $\left\langle\eta_{I}\left(t+t^{\prime}\right)\right\rangle\langle\varepsilon(t)\rangle=0$ for all $t^{\prime}$, we find

$$
\hat{\mathbf{\Xi}}(\tau)=\mathbf{S}^{\tau} \hat{\mathbf{G}} \text {. }
$$

\section{Concluding remarks}

Since we have presented a considerable amount of mathematical details above, it is worthwhile to provide a short summary. In the model presented in [4, the entire evolution depends only on the parameters, $\mu, F, N_{0}$, and $M_{I J}$. Strictly speaking, the only stationary state corresponds to total extinction $(\vec{n}=0)$. But, if mutation is suppressed $(\mu=0)$, then, not only does the configuration space break up into many sectors, but there will also be non-trivial long-lived $\left(O\left(\mathrm{e}^{N_{0}}\right)\right.$ generations $)$ states. Referred to as "truly stationary states," these communities consist of a fixed number $(\mathcal{N} \geq 1)$ of species. Typically, the population of each species is $O\left(N_{0}\right)$. Provided we are not near a "critical point," the full distribution of these populations can be studied by a systematic approximation scheme, starting with a multivariate Gaussian, parametrized by its mean and covariance matrix. For each TSS, our theory predicts these parameters and thus, the full distribution. Specifically, from the set of parameters, $\mu, F, N_{0}$, and $M_{I J}$, we can compute $\vec{n}^{*}, \hat{\mathbf{H}}$, and $\mathbf{S}=\mathbf{1}-\boldsymbol{\Lambda}$ (equations 3.23.3.26). Then, at the lowest order in our approximation scheme, the mean $\bar{n}$ and the covariance matrix $\hat{\mathbf{G}}$ are given explicitly (equations 4.10 and 4.16,4.17). In addition to this "static" aspect of the steady state, we also presented two ways of characterizing the "dynamic" aspect. One is the full distribution of sizes of steps (changes in the populations in a single time step, denoted by $\vec{s}$ ). Well approximated by a Gaussian also, this distribution has zero mean and covariance $\hat{\mathrm{g}}$, which is given explicitly by equation (5.17). The other is the correlation of the steps with the populations just before the step (specified by the deviations from the average, $\vec{\varepsilon}$ ). Denoted by $\hat{\mathbf{C}}$, this correlation is given explicitly by equation (5.22). Finally, we have shown that there is excellent agreement between these analytical predictions and Monte Carlo simulations in ten typical TSS communities (data for three of which are shown in tables 1 and 2 and figures 1 and 2).

With a solid understanding of the steady-state properties of stable communities in the absence of mutations, our next goal is a study of quasi-steady states of populations with low mutation rates. To carry out a serious analysis with $\mu>0$, we must define the restrictions on $R$ carefully. Otherwise, it would be impossible to find a satisfactory solution to the equation

$$
\mathcal{P}^{*}\left(\vec{n}^{\prime}\right)=\sum R\left(\vec{n}^{\prime} \mid \vec{n}\right) \mathcal{P}^{*}(\vec{n})
$$

As in the case for $\mu=0$, we may seek an approximate solution, in the form of a product of a Gaussian distribution for the dominant species and exponential distributions for the minority mutants [16. Provided this program is successful, the next step would be to study the probability of this type of QSS having "dangerous" mutants at the higher 
order of $\mu$, which hopefully will lead to some understanding of the distribution of QSS lifetimes [12] and the presence of $1 / f$ noise in power spectral densities. Beyond this step, perhaps sophisticated renormalization-group techniques can be marshalled to account for the self-similar patterns displayed, as well as to identify universality classes for such behaviour. Needless to say, even for such a simple model of coevolution, much remains to be explored.

\section{Acknowledgments}

We acknowledge enlightening discussions with many colleagues, in particular, HW Diehl, BU Felderhof, K Jain, J Krug, AJ McKane, and L Schäfer. One of us (RKPZ) thanks HW Diehl for his hospitality at the University of Duisburg-Essen (Germany) where some of this work was carried out. This research is supported in part by the Alexander von Humboldt Foundation (Germany), Florida State University through the Center for Materials Research and Technology and the School of Computational Science and Information Technology, and grants from the US National Science Foundation (DMR0088451, DMR-0120310, and DMR-0240078).

\section{Appendix A. Mathematical Detail}

Starting from the general expression for a discrete-time Markov process,

$$
\mathcal{P}\left(\vec{n}^{\prime}, t+1\right)=\sum_{\vec{n}} R\left(\vec{n}^{\prime} \mid \vec{n}\right) \mathcal{P}(\vec{n}, t),
$$

we write the mean-field approximation for the evolution equation as

$$
\vec{n}^{\mathrm{MF}}(t+1)=\vec{f}\left(\vec{n}^{\mathrm{MF}}(t)\right),
$$

where the functional form of $\vec{f}$ is given by

$$
\vec{f}(\vec{n})=\sum_{\vec{n}^{\prime}} \vec{n}^{\prime} R\left(\vec{n}^{\prime} \mid \vec{n}\right) .
$$

Note that the components of $\vec{n}$ play the role of coordinates in equation (A.1) and take only non-negative integer values in models of population dynamics. However, there is no guarantee that $\vec{f}$ will be an integer in equation (A.2), so that we must allow $\vec{n}^{\mathrm{MF}}$ to be continuous variables (functions of $t$ ). As we will see below, our analysis is much simplified if we also assume $\vec{n}$ to be continuous.

Focusing only on simple fixed points (as opposed to fixed cycles involving two or more points) of the mean-field theory, we denote a FP by $\vec{n}^{*}$. (To keep the notation from being too cumbersome, we suppress the superscript ${ }^{\mathrm{MF}}$ and only keep in mind that $\vec{n}^{*}$ represents just $\mathcal{N}$ real numbers.) The equation for determining $\vec{n}^{*}$ is

$$
\vec{n}^{*}=\vec{f}\left(\vec{n}^{*}\right) .
$$

Anticipating the need for the associated stability matrix, we write it as

$$
\left.\mathbf{S} \equiv \vec{\nabla} \vec{f}\right|_{\vec{n}^{*}}
$$


where $\vec{\nabla}$ stands for derivatives with respect to $\vec{n}$. Explicitly, the matrix elements of $\mathbf{S}$ are

$$
S_{I}^{J}=\partial f_{I} /\left.\partial n_{J}\right|_{\vec{n}^{*}}
$$

clearly asymmetric in general. As in our earlier study, we restrict ourselves to stable fixed points, so that the real parts of the eigenvalues of $\mathbf{S}$ (denoted by $\mathbf{1}+\hat{\boldsymbol{\Lambda}}$ in [4]) should lie in the interval $(-1,1)$. Indeed, we restrict our attention here to isolated FP's far from others, so that no (real part of any) eigenvalue is close to unity.

Turning to the full stochastic problem, we consider a stationary distribution $\mathcal{P}^{*}(\vec{n})$, which satisfies

$$
\mathcal{P}^{*}\left(\vec{n}^{\prime}\right)=\sum_{\vec{n}} R\left(\vec{n}^{\prime} \mid \vec{n}\right) \mathcal{P}^{*}(\vec{n}) .
$$

Therefore, it is a right eigenvector of the matrix $R\left(\vec{n}^{\prime} \mid \vec{n}\right)$ with unit eigenvalue. Its existence is guaranteed (though not necessarily its uniqueness, in general) by the presence of a left eigenvector of unit eigenvalue (i.e., $u(\vec{n})=1 \forall \vec{n}$ ), thanks to having probability conserving rates. If $\mathcal{P}^{*}$ is known, then we can compute the stationary averages of all quantities via equation (4.3). A different approach is to study the equations satisfied by these averages. Multiplying equation (A.7) by a quantity and summing, we find, in general, no closed equation. Instead, a tangle of infinitely many coupled equations appear. Examples are

$$
\left\langle n_{I}\right\rangle^{*}=\left\langle f_{I}(\vec{n})\right\rangle^{*} \quad \text { and } \quad\left\langle n_{I} n_{J}\right\rangle^{*}=\left\langle H_{I J}(\vec{n})\right\rangle^{*}
$$

where $f_{I}$ is an element of $\vec{f}$, given by equation (A.3), and

$$
H_{I J}(\vec{n}) \equiv \sum_{\vec{n}^{\prime}} n_{I}^{\prime} n_{J}^{\prime} R\left(\vec{n}^{\prime} \mid \vec{n}\right)
$$

In both cases, the right-hand sides contain expectation values of all powers of $\vec{n}$, so that infinitely many equations are needed to close the set.

In practice, for generic situations such as those described in the main text, we may approximate $\mathcal{P}^{*}$ by a Gaussian:

$$
\mathcal{P}^{*}(\vec{n}) \cong \mathcal{P}_{\mathrm{G}}(\vec{n})=\left(2 \pi N_{0}\right)^{-\mathcal{N} / 2}(\operatorname{det} \hat{\boldsymbol{\Gamma}})^{1 / 2} \exp \left[-\frac{1}{2 N_{0}}\left(n_{I}-\bar{n}_{I}\right) \hat{\Gamma}^{I J}\left(n_{J}-\bar{n}_{J}\right)\right],
$$

where the unknowns $\bar{n}_{I}$ and $\hat{\Gamma}^{I J}$ are to be determined. Naturally, we expect that the center of the Gaussian lies close to the mean-field FP, $\vec{n}^{*}$. This scenario is corroborated by our case studies above and our analysis below. Indeed, this approximation can be used as the starting point of a systematic expansion, which relies on having a large parameter $\left(N_{0}\right)$ controlling the fluctuations to $O\left(\sqrt{N_{0}}\right)$. In this context, $\bar{n}$ and $\hat{\boldsymbol{\Gamma}}$ are assumed to be $O\left(N_{0}\right)$ and $O(1)$, respectively. In addition to playing the role of ordering a systematic expansion, a large $N_{0}$ (and so, large $\vec{n}^{*}$ ) provides two further simplifications: (i) $\vec{n}$ may be promoted to continuous variables so that sums can be replaced by integrals, and (ii) integration can be extended to $[-\infty, \infty]$ while only incurring errors of $O\left(\mathrm{e}^{-\sqrt{N_{0}}}\right)$. 
Once $\bar{n}_{I}$ and $\hat{\Gamma}^{I J}$ are determined, then we can set up expansions for the averages of any quantity $Q$ :

$$
\langle Q(\vec{n})\rangle^{*} \equiv \sum_{\vec{n}} Q(\vec{n}) \mathcal{P}^{*}(\vec{n})
$$

starting with

$$
\int Q(\vec{n}) \mathcal{P}_{\mathrm{G}}(\vec{n}) \mathrm{d} \vec{n}
$$

Given that $n_{I}$ does not deviate far from $\bar{n}_{I}$, such integrals can be handled by first expanding $Q$ around $\bar{n}_{I}$ :

$$
Q(\vec{n})=\bar{Q}+Q^{I}\left(n_{I}-\bar{n}_{I}\right)+\frac{1}{2} Q^{I J}\left(n_{I}-\bar{n}_{I}\right)\left(n_{J}-\bar{n}_{J}\right)+\ldots,
$$

where the summation convention is used and

$$
\begin{aligned}
\bar{Q} & \left.\equiv Q(\vec{n})\right|_{\vec{n}=\bar{n}}, \\
Q^{I} & \left.\equiv \frac{\partial Q(\vec{n})}{\partial n_{I}}\right|_{\vec{n}=\bar{n}}, \\
Q^{I J} & \left.\equiv \frac{\partial^{2} Q(\vec{n})}{\partial n_{I} \partial n_{J}}\right|_{\vec{n}=\bar{n}} .
\end{aligned}
$$

The integral can now be performed, so that

$$
\langle Q(\vec{n})\rangle^{*} \cong \int Q(\vec{n}) \mathcal{P}_{\mathrm{G}}(\vec{n}) \mathrm{d} \vec{n}=\bar{Q}+\frac{N_{0}}{2} Q^{I J} \hat{G}_{I J}+\ldots,
$$

where $\hat{\mathbf{G}}=\hat{\boldsymbol{\Gamma}}^{-1}$. There is no need to be alarmed at the factor $N_{0}$ in the second term. Since $Q^{I J}$ involves two derivatives with respect to $\vec{n}$, it is typically of the order of $1 / N_{0}^{2}$ compared to $\bar{Q}$. Thus, the second term in this expansion is $O\left(1 / N_{0}\right)$ compared to the first.

With this machinery, we seek equations to fix $\bar{n}_{I}$ and $\hat{\mathbf{G}}$. From equation (4.4), we have, with $Q=f_{I}$,

$$
\bar{n}_{I}=\left\langle f_{I}(\vec{n})\right\rangle^{*} \cong f_{I}(\bar{n})+\frac{N_{0}}{2} f_{I}^{J K} \hat{G}_{J K}+\ldots .
$$

Again, we expect the second term to be $O\left(1 / N_{0}\right)$ compared to the first, so that we may write an expansion for $\bar{n}_{I}$ :

$$
\bar{n}_{I}=\bar{n}_{I}^{(0)}+\bar{n}_{I}^{(1)}+\ldots .
$$

Inserting this back into equation (A.18) and noting equation (A.4), we find the expected

$$
\bar{n}_{I}^{(0)}=n_{I}^{*}
$$

Proceeding to the next order, we find

$$
\bar{n}_{I}^{(1)}=f_{I}^{J}\left(\bar{n}_{I}^{(0)}\right) \bar{n}_{I}^{(1)}+\frac{N_{0}}{2} f_{I}^{J K} \hat{G}_{J K}
$$

where all functions of $\vec{n}$ in the last term can be evaluated at $\bar{n}_{I}^{(0)}$. To save notation, we will just write $\hat{G}_{J K}$ in lieu of the more explicit form: $\hat{G}_{J K}^{(0)}$. We recognize that $f_{I}^{J}\left(\bar{n}_{I}^{(0)}\right)$ is just the stability matrix $S_{I}^{J}$ and write

$$
\bar{n}_{I}^{(1)}=\frac{N_{0}}{2} V_{I}^{M} f_{M}{ }^{J K} \hat{G}_{J K}
$$


where

$$
\mathbf{V} \equiv(\mathbf{1}-\mathbf{S})^{-1}
$$

(the inverse of $-\tilde{\Lambda}$ in [4]). Let us emphasize that $V_{I}^{M}$ and $\hat{G}_{J K}$ are supposedly $O(1)$, while $f_{M}{ }^{J K}$ is $O\left(1 / N_{0}\right)$ so that the right-hand side of equation (A.22) is a quantity of $O(1)$. This expression also provides a precise meaning to the phrase "no eigenvalue (of $\mathbf{S})$ is close to unity," which appeared as a restriction on the FP's we study.

Extending this technique to $n_{I} n_{J}$, we consider

$$
N_{0} \hat{G}_{I J}+\left\langle n_{I}\right\rangle^{*}\left\langle n_{J}\right\rangle^{*}=\left\langle n_{I} n_{J}\right\rangle^{*}=\left\langle H_{I J}(\vec{n})\right\rangle^{*},
$$

from equations (4.4) and (A.8). Again, using the Gaussian approximation for both sides, we arrive at

$$
N_{0} \hat{G}_{I J}+\bar{n}_{I} \bar{n}_{J} \cong H_{I J}(\bar{n})+\frac{N_{0}}{2} H_{I J}{ }^{K M} \hat{G}_{K M}+\ldots
$$

At the lowest order, $O\left(N_{0}^{2}\right)$, of this equation, internal consistency will ensure that $H_{I J}\left(\bar{n}^{(0)}\right) \cong \bar{n}_{I}^{(0)} \bar{n}_{J}^{(0)}$ and should provide a check for tedious, error-prone computations. Furthermore, we can consider the difference:

$$
H_{I J}(\vec{n})-f_{I}(\vec{n}) f_{J}(\vec{n})
$$

and find that it is often one order lower (as explicitly shown in the main text for our model). In other words, we have

$$
H_{I J}\left(\vec{n}^{*}\right)-f_{I}\left(\vec{n}^{*}\right) f_{J}\left(\vec{n}^{*}\right) \sim O\left(N_{0}\right) .
$$

To emphasize this property, we explicitly extract a factor $N_{0}$ and define

$$
\hat{H}_{I J}(\vec{n}) \equiv\left[H_{I J}(\vec{n})-f_{I}(\vec{n}) f_{J}(\vec{n})\right] / N_{0},
$$

so that

$$
H_{I J}(\bar{n})=f_{I}(\bar{n}) f_{J}(\bar{n})+N_{0} \hat{H}_{I J}(\bar{n})
$$

Since we keep only terms of orders $N_{0}^{2}$ and $N_{0}$, we can evaluate $\hat{H}_{I J}(\bar{n})$ at $\vec{n}^{*}$ and define, for simplicity,

$$
\hat{H}_{I J} \equiv \hat{H}_{I J}\left(\vec{n}^{*}\right)
$$

As a reminder, this quantity is of $O(1)$. Next, to lowest order needed (i.e., $O(1)$ ), we find

$$
H_{I J}{ }^{K M}=S_{I}^{K} S_{J}^{M}+S_{I}^{M} S_{J}^{K}+f_{I} f_{J}^{K M}+f_{I}{ }^{K M} f_{J},
$$

all evaluated at the FP. Inserting these expressions into equation (A.25), we obtain

$$
\begin{aligned}
N_{0} G_{I J}+\bar{n}_{I} \bar{n}_{J} \cong & f_{I}(\bar{n}) f_{J}(\bar{n})+N_{0} \hat{H}_{I J} \\
& +\frac{N_{0}}{2}\left[S_{I}^{K} S_{J}^{M}+S_{I}^{M} S_{J}^{K}+f_{I} f_{J}^{K M}+f_{I}^{K M} f_{J}\right] G_{K M}+\ldots .
\end{aligned}
$$

Exploiting equation (A.18), we arrive at an equation for $\hat{\mathbf{G}}$ :

$$
\hat{G}_{I J}=\hat{H}_{I J}+\frac{1}{2}\left[S_{I}^{K} S_{J}^{M}+S_{I}^{M} S_{J}^{K}\right] \hat{G}_{K M} .
$$


But, $\mathbf{G}$ is symmetric, so that the final equation, in matrix form, reads

$$
\hat{\mathbf{G}}-\mathbf{S} \hat{\mathbf{G}} \mathbf{S}^{T}=\hat{\mathbf{H}} \text {. }
$$

Here, $\mathbf{S}^{T}$ denotes the transpose of $\mathbf{S}$, and, from equation (A.9), $\hat{\mathbf{H}}$ is necessarily symmetric. Again, let us emphasize that all quantities in this equation are $O(1)$. Unfortunately, there is no simple way to express $\hat{\mathbf{G}}$ in terms of the known matrices: $\mathbf{S}$ and $\hat{\mathbf{H}}$. A formal series can be written as

$$
\hat{\mathbf{G}}=\hat{\mathbf{H}}+\mathbf{S} \hat{\mathbf{H}} \mathbf{S}^{T}+\mathbf{S S} \hat{\mathbf{H}} \mathbf{S}^{T} \mathbf{S}^{T}+\ldots,
$$

equivalent to a recursion relation,

$$
\hat{\mathbf{G}}_{n}=\hat{\mathbf{H}}+\mathbf{S} \hat{\mathbf{G}}_{n-1} \mathbf{S}^{T}
$$

with $\hat{\mathbf{G}}_{0}=\hat{\mathbf{H}}$. It was this recursion method that was used to obtain numerical results for comparison with the Monte Carlo results in the figures and tables. Convergence to four significant digits was obtained with $n=20$ for $\mathcal{N}=2, n=30$ for $\mathcal{N}=3$, and $n=140$ for $\mathcal{N}=4$.

An explicit form for $\hat{\mathbf{G}}$ can be found when we examine this equation in the frame where $\mathbf{S}$ is diagonal. To be explicit, take matrix elements of equation (A.34) with $u_{a}^{I}$, the left eigenvectors of $\mathbf{S}$, which satisfy

$$
u_{a}^{I} S_{I}^{K}=\lambda_{a} u_{a}^{I} \quad(\text { no sum on } a) .
$$

The result is

$$
\hat{G}_{a b}=\hat{H}_{a b} /\left(1-\lambda_{a} \lambda_{b}\right) \quad(\text { no sum }),
$$

where

$$
\hat{G}_{a b}=\hat{G}_{I J} u_{a}^{I} u_{b}^{J} \quad \text { and } \quad \hat{H}_{a b}=\hat{H}_{I J} u_{a}^{I} u_{b}^{J} .
$$

To find the original matrix elements, we apply the dual set $v_{I}^{a}$ (i.e., the right eigenvectors of $\mathbf{S}$, normalized by $v_{I}^{a} u_{b}^{I}=\delta_{b}^{a}$ ) and obtain

$$
\hat{G}_{I J}=v_{I}^{a} v_{J}^{b} \hat{G}_{a b}=\sum_{a, b} v_{I}^{a} v_{J}^{b} \hat{H}_{a b} /\left(1-\lambda_{a} \lambda_{b}\right)
$$

Finally, this expression again shows the importance of insisting that "no eigenvalue (of $\mathbf{S}$ ) is close to unity." In case one or more $\lambda$ approaches unity, the system would be labelled "critical" or "multi-critical," in the language of phase transitions. Away from such points, the Gaussian approximation, along with its associated Ornstein-Uhlenbeck process, is adequate. For further details, see, for example, the books by van Kampen [15] and Risken [17].

\section{References}

[1] Drossel B 2001 Adv. Phys. 50209

[2] Lässig M and Valleriani A 2002 Biological Evolution and Statistical Physics (Berlin: SpringerVerlag) 
[3] Rikvold P A and Zia R K P 2003, in Computer Simulation Studies in Condensed Matter Physics $X V I$, ed D P Landau, S P Lewis and H-B Schüttler (Berlin: Springer-Verlag, in press) Preprint arXiv:nlin.AO/0303010

[4] Rikvold P A and Zia R K P 2003 Phys. Rev. E 68031913

[5] Hall M, Christensen K, di Collobiano S A and Jensen H J 2002 Phys. Rev. E 66011904

[6] Christensen K, di Collobiano S A, Hall M and Jensen H J 2002 J. theor. Biol. 21673

[7] di Collobiano S A, Christensen K and Jensen H J 2003 J. Phys. A 36883

[8] Bak P and Sneppen K 1993 Phys. Rev. Lett. 714083

[9] Gould S J and Eldredge N 1977 Paleobiology 3115

[10] Gould S J and Eldredge N 1993 Nature 366223

[11] Newman C M, Cohen J E and Kipnis C 1985 Nature 315400

[12] A similar distribution has been reported in Krug J and Karl C 2003 Physica A318 137. Recast as a distribution of inverse lifetimes (i.e., "decay rates"), $p(\tau \rightarrow \infty) \sim 1 / \tau^{2}$ becomes $p(r \rightarrow 0) \sim$ const., a property which appears not only plausible but also "generic" or "universal."

[13] Verhulst P F 1838 Corres. Math. et Physique 10113

[14] See, for example, Mohling F 1982 Statistical Mechanics, Method and Application (New York: Wiley); or Dorfman J R 1999 An introduction to Chaos in Nonequilibrium Statistical Mechanics (Cambridge: Cambridge Univ. Press)

[15] See, for example, Chapter IX in van Kampen N 1981 Stochastic Processes in Physics and Chemistry (Amsterdam: North Holland). A treatment which closely parallels the one given here can be found in Howard M and Zia R K P 2001 Int. J. Mod. Phys. B 15391

[16] Zia R K P and Rikvold P A, in preparation

[17] Risken H 1984 The Fokker-Planck Equation (Berlin: Springer-Verlag) 